\title{
ESTADO NUTRICIONAL Y PREFERENCIAS ALIMENTARIAS DE UNA POBLACIÓN DE ESTUDIANTES UNIVERSITARIOS EN BOGOTÁ
}

\author{
ESPERANZA FAJARDO ${ }^{1}$, YEIMI CAMARGO², ERIKA BUITRAGO ${ }^{3}$, \\ LEIDY PEÑA ${ }^{3}$, LINA RODRÍGUEZ ${ }^{3}$ \\ ${ }^{1}$ Nutricionista Dietista, Magister en Salud Pública, Profesora Asociada \\ Facultad de Medicina Universidad Militar Nueva Granada \\ ${ }^{2}$ Médico \\ Joven Investigadora Grupo CATCH \\ ${ }^{3}$ Estudiantes Facultad de Medicina. Universidad Militar Nueva Granada \\ Correspondencia: esperanza.fajardo@unimilitar.edu.co
}

Recibido: 8 Enero 2016 Aceptado: 6 Julio 2016

\begin{abstract}
RESUMEN
Introducción: Durante la vida universitaria los estudiantes deberían fortalecer los hábitos de alimentación saludables, lo cual contribuirá al bienestar en la vida del profesional adulto. El objetivo de este estudio fue evaluar el estado nutricional y las preferencias alimentarias en un grupo de estudiantes universitarios de pregrado.
\end{abstract}

Materiales y métodos: Se realizó un estudio transversal. Fueron incluidos los estudiantes que aceptaron participar en el estudio y que completaron una única encuesta que incluía aspectos sobre consumo actual de alimentos en la Universidad, características generales, datos antropométricos y propiedades organolépticos de los alimentos. Los datos se analizaron con el programa SPSS versión 20.

Resultados: 181 estudiantes con edad entre 16 y 25 años completaron el cuestionario. El 83,6 \% presentó un índice de masa corporal - IMC normal, 7,9 \% bajo peso, 7,3 \% sobrepeso y 1,1\% obesidad. No se encontró asociación entre el estado nutricional y las personas con las que vive el estudiante $(\mathrm{p}=0,991)$. El 17,6 \% refirió tomar bebidas energéticas y el $41,5 \%$ bebidas gaseosas al menos una vez por semana. El 37,5\% demostró escoger comidas rápidas al menos una vez por semana. El consumo diario de productos lácteos, frutas y verduras fue observado en menos del $20,0 \%$ de los estudiantes.

Conclusiones: Los hallazgos demuestran la importancia de establecer tempranamente intervenciones para alcanzar hábitos de alimentación saludables. Esas iniciativas podrían ser más exitosas si incluyen el ámbito familiar, social y las instituciones educativas.

Palabras Clave: Estado nutricional, Consumo de alimentos, Estudiantes, Calidad de los alimentos. 


\title{
NUTRITIONAL STATUS AND FOOD PREFERENCES AMONG UNIVERSITY STUDENTS IN BOGOTA
}

\begin{abstract}
Introduction: During University life students should strengthen healthy food habits which will contribute to the well-being of the professional adult life. The aim of this study was to assess the nutritional status and the food preferences of a group of undergraduate university students.
\end{abstract}

Materials and methods: A cross-sectional study was realized. It included students who accepted to participate and completed a single, self-administered food habits questionnaire that asked about their current food practices at the university, general characteristics, anthropometric data, and food organoleptic properties. The data were analyzed with SPSS version 20.

Results: One hundred eighty one students aged $16-25$ completed the questionnaire. The 83,6\% had normal BMI, $7,9 \%$ undernourishment, $7,3 \%$ overweight and $1,1 \%$ obesity. No association between the people with whom the student lives and nutritional status was found $(p=0,991)$. The $17,6 \%$ took energy drinks and $41.5 \%$ sodas at least once per week. The 37,5\% showed to choose junk food at least once per week. Daily intake of dairy foods, fruits and vegetables was observed in less of $20 \%$ of the students.

Conclusions: The findings show the importance of establishing early interventions for reaching healthy habits of feeding. These initiatives could be more successful if they include the familiar and social environment and educational institutions.

Key Words: Nutritional Status, Food consumption, Students, Food quality.

\section{ESTATUTO NUTRICIONAL E PREFERÊNCIAS ALIMENTARES ENTRE ESTUDANTES UNIVERSITÁRIOS EM BOGOTÁ}

\section{RESUMO}

Introdução: Durante a vida universitária os alunos devem fortalecer os hábitos alimentares saudáveis que contribuirão para o bem-estar da vida adulta profissional. O objetivo deste estudo foi avaliar o estado nutricional e as preferências alimentares de um grupo de estudantes universitários de graduação.

Materiais e métodos: Foi realizado um estudo transversal. Ele incluía estudantes aceitaram participar e que completaram um único, questionário auto-administrado que os hábitos alimentares questionados sobre as suas práticas atuais de alimentos na universidade, características gerais, dados antropométricos e organoléptica alimentos você propriedades. Os dados foram analisados com SPSS versão 20.

Resultados: Cento e oitenta e um alunos de 16 a 25 anos completaram o questionário. Os 83,6\% apresentaram IMC normal, 7,9\% desnutrição, 7,3\% sobrepeso e 1,1\% obesidade. Não foi encontrada associação entre as pessoas com as quais o aluno vive e o estado nutricional ( $p=0,991)$. Os 17,6\% tomaram bebidas energéticas e 41,5\% de refrigerantes pelo menos uma vez por semana. Os 37,5\% mostraram escolher junk food pelo menos uma vez por semana. A ingestão diária de alimentos lácteos, frutas e verduras foi observada em menos de $20 \%$ dos alunos.

Conclusões: The findings show the importance of establishing early interventions for reaching healthy habits of feeding. Estas iniciativas poderiam ser mais bem sucedidas se incluírem o ambiente familiar e social e as instituições educacionais.

palavras-chave: Estado nutricional, Consumo de comida, Estudantes, Qualidade alimentar. 


\section{Introducción}

En las últimas décadas la población ha cambiado sus hábitos de vida, los cuales no siempre han sido favorables para la salud. La preferencia por alimentos de bajo valor nutricional, el sedentarismo, el estrés y el consumo de sustancias como el alcohol y el tabaco, son algunas de las causas de múltiples enfermedades como el sobrepeso, la obesidad, la hipercolesterolemia, hipertensión, entre otras (1). La prevalencia de la obesidad ha venido en aumento, presentándose cada vez más, desde etapas tempranas de la vida, contribuyendo al riesgo de enfermedades crónicas como la enfermedad cerebro vascular $y$ enfermedades coronarias a nivel mundial $(2,3,4)$.

La Organización Mundial de la Salud (OMS) ha hecho énfasis en la importancia de la alimentación adecuada para el crecimiento físico, el desarrollo mental, el desempeño, la productividad, la salud y el bienestar en todas las etapas de la vida del ser humano (5). En este contexto, las características organolépticas de los alimentos, entendidas como la impresión de éstos por medio de los órganos de los sentidos al percibir sus características (color, olor, tamaño, forma, sabor y textura), generan ciertas preferencias hacia algunos alimentos específicos, afectando positiva o negativamente la salud de las personas (6).

Los hábitos alimentarios pueden verse reflejados en el estado de salud de los jóvenes, en su rendimiento académico y en su vida profesional y social futura $(1,6,7)$. Por esta razón, en este estudio se busca describir las características nutricionales y las preferencias alimentarias de un grupo de universitarios y las propiedades organolépticas de los alimentos a los cuales tienen acceso en su sitio de estudio de la ciudad de Bogotá.

\section{Materiales y Métodos}

Estudio descriptivo transversal. La población estudio correspondió a estudiantes de primero a sexto semestre de medicina. La muestra fue de 181 sujetos quienes aceptaron voluntariamente participar en el estudio, de los cuales el 64,1 \% fueron mujeres. Los datos fueron recolectados durante el segundo periodo académico del año 2015.

Se aplicó un cuestionario previamente validado con información de aspectos sociodemográficos, antropométricos (peso y talla), frecuencia de consumo de los alimentos ofrecidos en la cafetería de la facultad y evaluación de las propiedades organolépticas de dichos alimentos. La sección "Frecuencia de Consumo de Alimentos" fue organizada por grupos: Leche o derivados, frutas, verduras, cereales y productos derivados, grasas, dulces y golosinas, bebidas calientes y frías. Además se incluyó comidas elaboradas como el desayuno, almuerzo casero, sopas y comidas rápidas.

Se solicitó a los estudiantes calificar las propiedades organolépticas de los alimentos tales como olor, sabor, apariencia y textura en una escala de 1 "muy desagradable" a 5 "muy agradable" (8).

Por último los estudiantes calificaron la calidad de los productos ofrecidos en la cafetería como excelente, muy bueno, bueno, regular y malo.

Se calculó el índice de masa corporal (IMC) a partir de los datos de peso y talla (Peso/Talla ${ }^{2}$ ) expresado en $\mathrm{kg} /$ $\mathrm{m}^{2}$. Para la clasificación nutricional, se utilizaron las tablas de IMC por la edad para hombres y mujeres de 2 a 20 años establecidas por el Centro de Estadísticas de Salud de Estados Unidos (USA National Center for Health Statistics). Los valores de índice de masa corporal $<5^{\circ}$ percentil son considerados como bajo peso; entre el $5^{\circ}$ percentil y $<85^{\circ}$ percentil son definidos como normal; sobrepeso entre $\geq 85^{\circ}$ percentil y $<95^{\circ}$ percentil, y obesidad $\geq 95^{\circ}$ percentil (9).

Los datos se registraron en planillas de Microsoft Excel y el análisis estadístico se realizó con el programa SPSS versión 20 (SPSS Inc. Chicago, Illinois USA). Se realizó análisis descriptivo a través de tablas de distribución de frecuencias para variables cualitativas y medidas de tendencia central para variables cuantitativas. Para evaluar asociación entre variables cualitativas se usó la prueba de Chi cuadrado.

\section{Resultados}

De los estudiantes incluidos en este estudio, 116 fueron mujeres y 65 hombres con un promedio de edad de 19,2 $\pm 1,6$ años (rango de 16 a 25).

El 7,3\% de los 181 estudiantes, presentó sobrepeso y el 1,12\% obesidad. Se observó que el 16,3\% de los sujetos presentó alguna alteración nutricional según el IMC. No se encontraron diferencias en el estado nutricional de los estudiantes de acuerdo al semestre cursado (Tabla 1). 
Tabla 1. Estado nutricional de los estudiantes por semestre académico

\begin{tabular}{|c|c|c|c|c|c|c|c|c|c|c|}
\hline Semestre & \multicolumn{2}{|c|}{ Bajo peso } & \multicolumn{2}{c|}{ Peso normal } & \multicolumn{2}{c|}{ Sobre peso } & \multicolumn{2}{c|}{ Obesidad } & \multicolumn{2}{c|}{ Total } \\
\hline & $\mathrm{N}$ & $\%$ & $\mathrm{~N}$ & $\%$ & $\mathrm{~N}$ & $\%$ & $\mathrm{~N}$ & $\%$ & $\mathrm{~N}$ & $\%$ \\
\hline $\mathbf{1}$ & 6 & $42,9 \%$ & 27 & $18,2 \%$ & 1 & $7,7 \%$ & 1 & $50 \%$ & 35 & $19,8 \%$ \\
\hline $\mathbf{2}$ & 2 & $14,3 \%$ & 48 & 32,45 & 6 & $46.2 \%$ & 0 & $0 \%$ & 56 & $31,6 \%$ \\
\hline $\mathbf{3}$ & 1 & $7,1 \%$ & 18 & $12,2 \%$ & 1 & $7,7 \%$ & 1 & $50 \%$ & 21 & $11,9 \%$ \\
\hline $\mathbf{4}$ & 2 & $14,3 \%$ & 5 & $3,4 \%$ & 1 & $7,7 \%$ & 0 & $0 \%$ & 8 & $4,5 \%$ \\
\hline $\mathbf{5}$ & 0 & $0 \%$ & 24 & $16,2 \%$ & 0 & $0 \%$ & 0 & $0 \%$ & 24 & $13,6 \%$ \\
\hline $\mathbf{6}$ & 3 & $21,4 \%$ & 26 & $17,6 \%$ & 4 & $30,8 \%$ & 0 & $0 \%$ & 33 & $18,6 \%$ \\
\hline Total & $\mathbf{1 4}$ & $\mathbf{1 0 0} \%$ & $\mathbf{1 4 8}$ & $\mathbf{1 0 0} \%$ & $\mathbf{1 3}$ & $\mathbf{1 0 0 \%}$ & $\mathbf{2}$ & $\mathbf{1 0 0 \%}$ & $\mathbf{1 7 7}$ & $\mathbf{1 0 0 \%}$ \\
\hline
\end{tabular}

Fuente: elaboración propia.

El 83,6\% de los estudiantes vive con sus padres y/o hermanos y el 51,4 \% acostumbra a consumir la mayor parte de las comidas principales con los mismos familiares. El 93,0 \% de los sujetos que presenta alguna alteración nutricional según el IMC vive con los padres y/o hermanos y el 7,0 \% restante refiere vivir solos. No se encontró asociación entre estado nutricional y personas con las que vive el estudiante $(\mathrm{p}=0,991)$. (Tabla 2$)$.

Tabla 2. Relación entre el Índice de Masa corporal y convivencia.

\begin{tabular}{|l|c|c|c|c|c|c|c|c|c|c|}
\hline \multicolumn{1}{|c|}{ Semestre } & \multicolumn{2}{c|}{ Bajo peso } & \multicolumn{2}{c|}{ Peso normal } & \multicolumn{2}{c|}{ Sobre peso } & \multicolumn{2}{c|}{ Obesidad } & \multicolumn{2}{c|}{ Total } \\
\hline & $\mathrm{N}$ & $\%$ & $\mathrm{~N}$ & $\%$ & $\mathrm{~N}$ & $\%$ & $\mathrm{~N}$ & $\%$ & $\mathrm{~N}$ & $\%$ \\
\hline Padres y/o hermanos & 13 & $92,9 \%$ & 121 & $81,8 \%$ & 12 & $92,3 \%$ & 2 & $100 \%$ & 148 & $83,6 \%$ \\
\hline Otros familiares & 0 & $0 \%$ & 12 & $8,1 \%$ & 0 & $0 \%$ & 0 & $0 \%$ & 12 & $6,8 \%$ \\
\hline Amigos & 0 & $0 \%$ & 2 & $1,4 \%$ & 0 & $0 \%$ & 0 & $0 \%$ & 2 & $1,1 \%$ \\
\hline Solo & 1 & $7,1 \%$ & 12 & $8,1 \%$ & 1 & $7,7 \%$ & 0 & $0 \%$ & 14 & $7,9 \%$ \\
\hline Otros & 0 & $0 \%$ & 1 & $7 \%$ & 0 & $0 \%$ & 0 & $0 \%$ & 1 & $6 \%$ \\
\hline Total & $\mathbf{1 4}$ & $\mathbf{1 0 0 \%}$ & $\mathbf{1 4 8}$ & $\mathbf{1 0 0 \%}$ & $\mathbf{1 3}$ & $\mathbf{1 0 0} \%$ & $\mathbf{2}$ & $\mathbf{1 0 0 \%}$ & $\mathbf{1 7 7}$ & $\mathbf{1 0 0 \%}$ \\
\hline
\end{tabular}

Fuente: elaboración propia.

Con relación a la frecuencia de consumo de alimentos y bebidas ofrecidos en la cafetería, se observó que el 19,6 $\%$ de los estudiantes refiere desayunar diariamente en la cafetería de la facultad mientras que el 47,8\% nunca lo hacen en la facultad. El 22,0 \% de los estudiantes adquiere diariamente el almuerzo en la cafetería y el 14,5 $\%$ de dos a tres veces por semana en este sitio.

En cuanto a la frecuencia del consumo de comidas rápidas (hamburguesas, perro caliente, similares), el 37.5 $\%$ del total de la población consume al menos una vez a la semana estos alimentos y un $23,0 \%$ asegura nunca consumir este tipo de alimento en la cafetería. El 34,3
\% refiere consumir ocasionalmente las comidas rápidas ofrecidas.

El 17,6 \% de los estudiantes acostumbra consumir bebidas energéticas al menos una vez a la semana y el 41,5 \% refiere tomar al menos una vez a la semana bebidas gaseosas.

El 46,2 \% de los estudiantes con bajo peso y el 30,8 \% que presentó sobrepeso refirieron el consumo ocasional de bebidas gaseosas en la cafetería de la facultad.

El 47,0 \% de los sujetos con exceso de peso refirió con- 
sumir comidas rápidas ocasionalmente, mientras que el $40,0 \%$ de este grupo nunca consume estos alimentos en la cafetería de la universidad. Así mismo, se observó que el 36,0 \% de los sujetos con exceso de peso acostumbra a consumir empaquetados al menos una vez a la semana. El 27,1\% de los estudiantes respondió nunca adicionarle salsas tales como mayonesa, salsa de tomate u otros aderezos a las comidas.

Más de la mitad (62.3\%) de la población consume dulces y golosinas al menos una vez por semana. Del total de sujetos con exceso de peso, el 50,0 \% refirió consumir ocasionalmente o nunca este tipo de alimentos.

Se destaca que menos del $20 \%$ de la población consume leche o sus derivados. El 44,5\% reportó consumir ocasionalmente o nunca jugos de fruta natural y el 54,8 $\%$ reportó el consumo de fruta entera con esa misma fre- cuencia. Menos del 20,0 \% refirió el consumo de frutas y verduras todos los días.

La evaluación por parte de los estudiantes de las características organolépticas de los alimentos ofrecidos en la cafetería de la facultad, reportó que la percepción olfativa de los alimentos tiende a ser indiferente; el olor de los productos de pastelería es agradable en mayor proporción que los demás alimentos evaluados (25\%), seguidos por las comidas rápidas (24\%). El 33\% de los estudiantes no opina con relación a la percepción olfativa de los vegetales. Un alto porcentaje de estudiantes demuestra indiferencia a esta propiedad organoléptica de los alimentos ofrecidos en la cafetería (Figura 1).

El 22,0 \% de los estudiantes opina que los almuerzos ofrecidos en la cafetería tienen sabor agradable y el 20,2 \% opina lo mismo de las comidas rápidas (Figura 2).

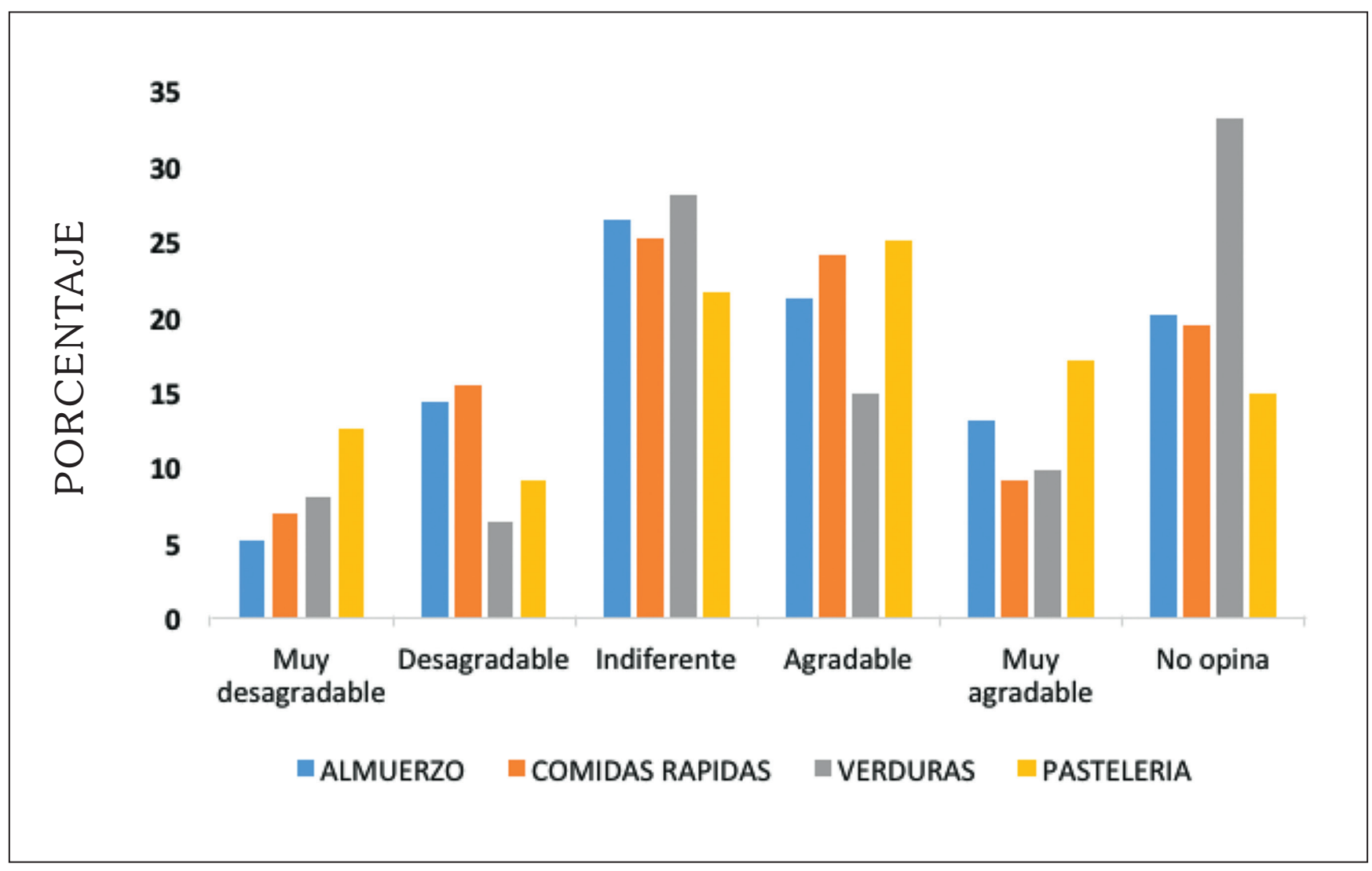

Figura 1. Percepción olfativa de los alimentos ofrecidos en la cafetería.

Fuente: elaboración propia. 


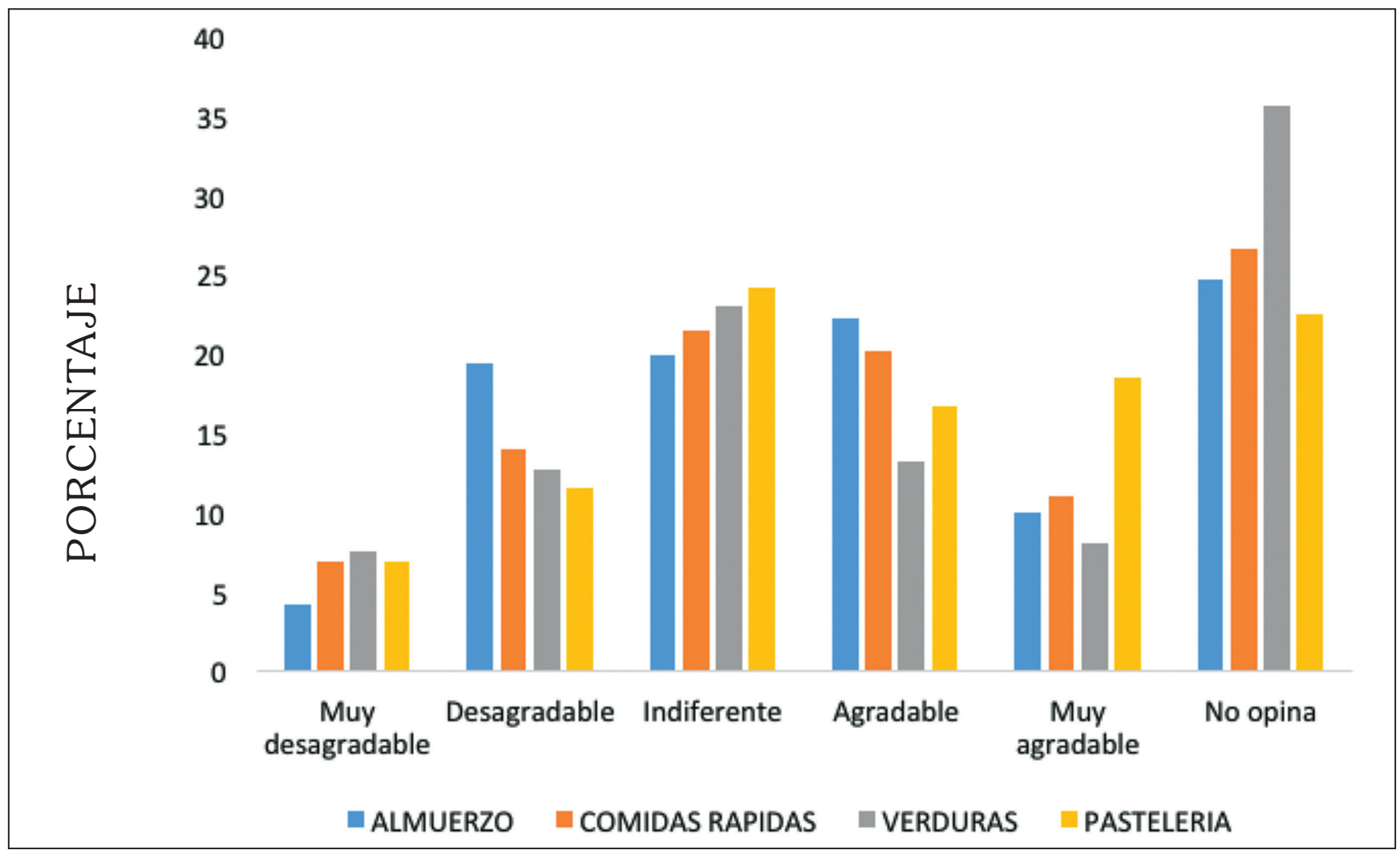

Figura 2. Percepción gustativa de los alimentos ofrecidos en la cafetería. Fuente: elaboración propia.

El 45,4\% de los estudiantes consideraron de apariencia entre agradable y muy agradable, a los productos de pastelería, panadería y similares. Sin embargo, se des- taca la tendencia a no opinar o ser indiferentes ante la apariencia del almuerzo, comidas rápidas y las verduras (Figura 3).

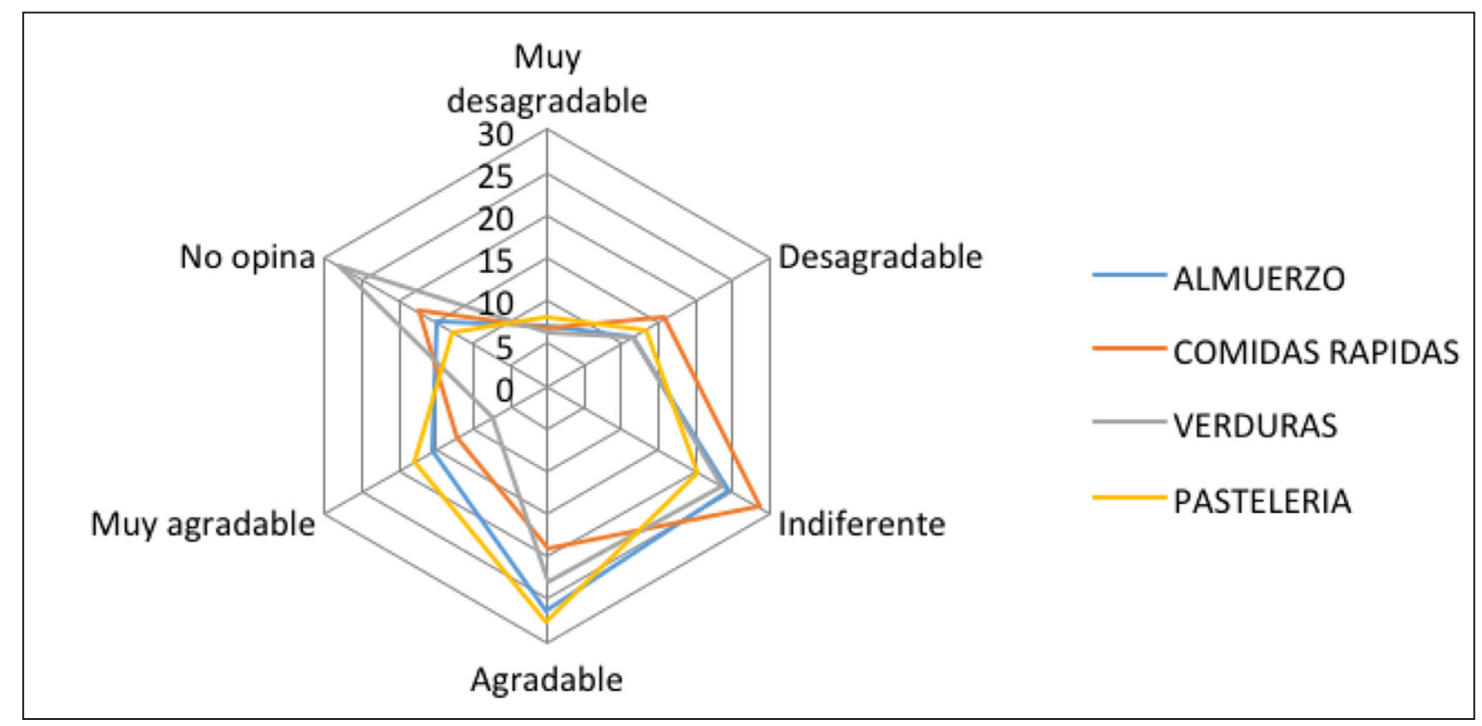

Figura 3. Percepción de la apariencia de los alimentos ofrecidos en la cafetería Fuente: elaboración propia. 


\section{Discusión}

En este estudio se encontró un menor porcentaje de exceso de peso en relación al reportado en otros estudios en grupo etario similar $(10,11)$. Sin embargo, con relación a la frecuencia de consumo de bebidas gaseosas y de comidas rápidas el porcentaje es similar al encontrado en la Encuesta Nacional de la Situación Nutricional de Colombia -ENSIN del año 2010 (10). El consumo de comidas rápidas es independiente del indice de masa corporal, hallazgo similar a estudios en países de altos ingresos (12).

Se observó una baja preferencia por productos lácteos, verduras y frutas enteras. Diferentes estudios realizados en universitarios concuerdan con estos resultados, en cuanto a una alimentación con poca selección de frutas y verduras y exclusión de alguna de las comidas principales $(7,13)$. Se ha descrito la importancia de identificar la cantidad y frecuencia en el consumo de un alimento, más que el tipo de alimento en sí como parte de la evaluación de los hábitos alimentarios (13).

Es conocido que las preferencias alimentarias aparecen desde edades tempranas de la vida y se ven influenciadas por el entorno. En la adolescencia los hábitos alimentarios suelen variar de acuerdo a otros factores, principalmente los relacionados con el círculo social, el tiempo que se disponga para tomar los alimentos y la oferta alimentaria de los sitios que se frecuentan $(13,14,15)$. En este estudio, se encontró que los estudiantes acostumbran a consumir bebidas energizantes al menos una vez a la semana. Se ha demostrado que los estudiantes que reportan el consumo de bebidas energéticas son $66 \%$ más propensos a la falta de atención e hiperactividad (11)

Dentro de la percepción de las características organolépticas de los alimentos, se evidenció que las comidas rápidas y la pastelería fueron mejor calificadas al considerarse que presentan mejor olor y sabor que las verduras ofrecidas en la cafetería de la facultad. Se ha encontrado que estas características son fundamentales en el momento de elegir un alimento y que la percepción del sabor es una barrera para el consumo de alimentos saludables como los vegetales (16). De otra parte, se ha descrito que la percepción olfatoria evoca emociones o recuerdos en las personas influyendo en la elección de los alimentos sin ser plenamente conscientes de ello y que dicha percepción va disminuyendo a medida que aumenta la edad (17). La elección y/o preferencias de ciertos alimentos están estrechamente relacionadas con las emociones/recuerdos que producen los alimentos y el gusto (17).

\section{Conclusiones}

Las preferencias alimentarias en esta población se asemejan a los encontrados en estudios similares. Se observa preferencia por alimentos de bajo valor nutricional, mayor consumo de alimentos de pastelería y comidas rápidas, siendo estas últimas conocidas por su densidad calórica, el alto contenido en grasas saturadas y colesterol. Los lácteos, las frutas y las verduras son los alimentos de menor consumo.

Las bebidas energizantes están presentes en la alimentación de estos universitarios, productos que cada vez son más consumidos por los jóvenes.

Es importante tener presente las características organolépticas de los alimentos para promover la adecuada elección de los mismos con mayor valor nutricional y que las personas sientan agrado por las comidas y no solamente una elección como parte de la necesidad de alimentarse.

Se requiere enfocar la atención en el diseño e implementación de actividades de promoción de la salud, autocuidado y prevención de enfermedades crónicas que incluyan estrategias para fortalecer hábitos alimentarios adecuados en la población juvenil con el fin de lograr en un futuro una generación más sana. Las intervenciones para promover una alimentación saludable podrían tener mayor éxito, si se incluyen los diferentes escenarios de la vida diaria incluidas las entidades educativas.

\section{Conflicto de intereses}

Los autores declaran no tener de manera directa o indirecta, algún tipo de conflicto de intereses financieros, académicos o laborales que puedan poner en peligro la validez de este estudio.

\section{Financiación}

Este trabajo fue aprobado y financiado por la Universidad Militar Nueva Granada. 


\section{Referencias}

1. Irazusta-stiazaran A, Hoyos-Cillero I, Díaz-Ereño E, Irazusta-Astiazaran J, Gil-Goikouria J, Gil-Orozko S. Alimentación de estudiantes universitarios. República Dominicana. Rev Osasunaz 2007; 8:7-18.

2. Allemand-Jander DI. Clinical diagnosis of metabolic and cardiovascular risks in overweight children: early development of chronic diseases in the obese child. International Journal of Obesity 2010; 34:32-36

3. Requejo A, Ortega R. Nutrición en la adolescencia y juventud. Madrid: Ed. Complutense; 2002. https://books. google.com.co/books?isbn=8474916917 (último acceso 23 de agosto 2016)

4. Guerra C, Vila J, Apolinaire J, Cabrera A, et al. Factores de riesgo asociados a sobrepeso y obesidad en adolescentes. MediSur 2009; 7(2): 25-34.

5. Organización Mundial de la Salud. Salud de la madre, el recién nacido, del niño y del adolescente. 2016. OMS. http://www.who.int/maternal_child_adolescent/topics/ child/development/es/ (ultimo acceso 23 de agosto 2016)

6. Gallardo-Wong I, Buen Abad-Eslava L. Mala nutrición en estudiantes universitarios de la Escuela de Dietética y Nutrición del ISSSTE. México. Rev Med UV 2011; 11 (1): 6-11.

7. Vargas-Zárate, Becerra-Bulla F, Prieto-Suárez E. Evaluación de la ingesta dietética en estudiantes universitarios. Bogotá, Colombia. Rev Salud Pública 2010; 12 (1):116-125.

8. Vikrant-Arya, Raneev-Thakur. Organoleptic and Microscopic Analysis of Gentiana regeliana. India. Journal of Pharmacognosy and Phytochemistry 2012;1 (2): 32.

9. Heymsfield SB, Lohman TG, Wang Z, Going SB. Composición Corporal. $2^{\circ}$ ed. México: Mc Graw Hill; 2005. p.521.

10. Ministerio de Protección Social, Instituto Colombiano de Bienestar Familiar, Instituto Nacional de Salud. Encuesta Nacional de la Situación Nutricional en Colombia 2010 ENSIN. Bogotá: Oficina Asesora de Comunicaciones y Atención al Ciudadano ICBF; 2011. p. 1-509, 6.
11. Pereira PF, Serrano HM, Carvalho GQ, Lamounier JA, Peluzio MG, Franceschini SC, et al. Body fat location and cardiovascular disease risk factors in overweight female adolescents and eutrophic female adolescents with a high percentage of body fat. Cardiology in the Young 2012; 22:162-169

12. De Vogli R, Kouvonen A, Gimeno D. La influencia de la desregulación del mercado en el consumo de comida rápida y el índice de masa corporal: un análisis de series temporales entre países. En: Boletín de la Organización Mundial de la Salud. 2014; 92 (2):77-152. Disponible en: www.who.int/bulletin/volumes/92/2/13-120287-ab/es/

13. Saad C, Ibáñez E, León C, Colmenares C, Vega N, Díaz $\mathrm{Y}$, et al. Cambios de los hábitos alimentarios de los estudiantes de enfermería de la Universidad El Bosque durante su proceso de formación académica, Bogotá, D.C., 2007. Rev. Colombiana de enfermería 2008; 3: 1909-1621.

14. Ferro RA, Maguiña VJ. Relación entre hábitos alimentarios e índice de masa corporal en estudiantes de una universidad pública según área de estudio. Lima, 2012, 65 h. Trabajo de grado (Nutrición). Universidad Nacional Mayor De San Marcos. Facultad de Medicina Humana. Disponible en:http://cybertesis.unmsm.edu.pe/bitstream/ cybertesis/1123/1/Ferro_mr.pdf

15. Montero Bravo A., Úbeda Martín N., García González A. Evaluación de los hábitos alimentarios de una población de estudiantes universitarios en relación con sus conocimientos nutricionales. Nutr. Hosp. 2006;21(4): 466-73.

16. Skuland E,. Healthy Eating and Barriers Related to Social Class. The case of vegetable and fish consumption in Norway. Appetitte 2015; 92:217-226.

17. Uijl LC., Jager G, Graaf C, Meiselman HL., Kremer S. Emotion, olfaction, and age: A comparison of self-reported food-evoked emotion profiles of younger adults, older normosmic adults, and older hyposmic adults. Food Quality and Preference 2015; 48:199-209. 